\title{
Information about the Weight of Grasped Objects from Vision and Internal Models Interacts within the Primary Motor Cortex
}

\author{
Morrison N. Loh, Louise Kirsch, John C. Rothwell, Roger N. Lemon, and Marco Davare \\ Sobell Department of Motor Neuroscience and Movement Disorders, Institute of Neurology, UCL, Queen Square, London WC1N 3BG, United Kingdom
}

When grasping and lifting different objects, visual cues and previously acquired knowledge enable us to prepare the upcoming grasp by scaling the fingertip forces according to the actual weight of the object. However, when no visual information is available, the weight of the object has to be predicted based on information learned from previous grasps. Here, we investigated how changes in corticospinal excitability (CSE) and grip force scaling depend on the presence of visual cues and the weight of previously lifted objects. CSE was assessed by delivering transcranial magnetic stimulation (TMS) at different times before grasp of the object. In conditions in which visual information was not provided, the size of motor evoked potentials (MEP) was larger when the object lifted was preceded by a heavy relative to a light object. Interestingly, the previous lift also affected MEP amplitude when visual cues about object weight were available but only in the period immediately after object presentation $(50 \mathrm{~ms})$; this effect had already declined for TMS delivered $150 \mathrm{~ms}$ after presentation. In a second experiment, we demonstrated that these CSE changes are used by the motor system to scale grip force. This suggests that the corticospinal system stores a "sensorimotor memory" of the grasp of different objects and relies on this memory when no visual cues are present. Moreover, visual information about weight interacts with this stored representation and allows the corticospinal system to switch rapidly to a different model of predictive grasp control.

\section{Introduction}

Initiation of grasp involves the coordination of both external sensory inputs and internal models of the desired movement. Sensory information related to the size, shape, and orientation of objects is provided through vision (Gordon et al., 1991; Jenmalm and Johansson, 1997). Internal models related to object weight are learned through previous motor experience and allow prediction of the actual grip force appropriate for the object load (Flanagan and Wing, 1997; Witney et al., 2000; Wolpert and Flanagan, 2001).

When lifting an object, we use a force that is precisely scaled to its weight. This accurate scaling of the lift force relies on predictions based on previous lifts or estimation of the weight of a particular object based on available visual information. When presented with an object of unknown weight, subjects will first use an inappropriate force, which is usually an overestimate of the actual force required to lift the object. Subjects adjust and refine the force needed during subsequent lifts of the same object to achieve the optimal force required. This rapid adaptation of force generation indicates that there is a real-time motor updat-

\footnotetext{
Received Dec. 15, 2009; revised Jan. 16, 2010; accepted Feb. 4, 2010.

M.N.L. received a Wellcome Trust scholarship. M.D. is funded by Wellcome Trust Grant WT083450. We thank Ed Bye and Victor Baller for the design of the turntable and manipulandum.

This article is freely available online through the J Neurosci Open Choice option.

Correspondence should be addressed to Dr. Marco Davare, Sobell Department of Motor Neuroscience and Movement Disorders, Institute of Neurology, UCL, Queen Square, London WC1N3BG, UK. E-mail: mdavare@ion.ucl.ac.uk. DOI:10.1523/JNEUROSCI.6207-09.2010

Copyright $\odot 2010$ the authors $\quad 0270-6474 / 10 / 306984-07 \$ 15.00 / 0$
}

ing of the information used to coordinate the grasp (Johansson and Westling, 1984, 1988).

Johansson and Westling (1988) first showed that information acquired while subjects grasped and lifted an object influenced the preparatory grip force used for a subsequent lift. More recently, Chouinard et al. (2005) found that the primary motor (M1) and dorsal premotor cortices played an important role in force scaling depending on the previous lift and on visual cues, respectively (Chouinard et al., 2005). Although M1 seems to be involved in storing, or recruiting from other areas, a "sensorimotor memory" about previous lifts, it is not known how this "memory" is represented in M1 and how visual information about weight, when it becomes available, interacts with this memory. Therefore, we investigated whether corticospinal excitability is influenced by the sensorimotor memory about the previous lift and how and when visual information about the weight of an object interacts with this memory of the previous lift.

The rationale for the present study was to examine corticospinal excitability (CSE) and grip lift performance for two pairs of drinking glasses: for one pair, the glass was transparent, providing a clear visual cue to whether the glass was full (heavy) or nearly empty (light); the other pair of glasses were identical, except that the glass was opaque and therefore its weight was unknown to the subject. We sought to analyze (1) the simple effect of sensorimotor memory by comparing the motor evoked potentials (MEPs) obtained when preparing to lift an object of unknown weight preceded by either a heavy or a light object, (2) the simple effect of visual cues by comparing lifts of visibly heavy versus visibly light objects, both preceded by lift of an object having the same weight, 
and (3) the interactions between visual input and sensorimotor memory by comparing trials of visible objects preceded by trials in which heavy or light objects were lifted.

\section{Materials and Methods}

\section{Subjects}

We recruited eight subjects for experiment 1 (five males; $24 \pm 4$ years old) and seven for experiment 2 (three males; $23 \pm 5$ years old; five of experiment 1). All subjects were right-handed according to the Edinburgh handedness inventory (Oldfield, 1971). Their vision was normal or corrected to normal, and none had a history of neurological disease. Subjects were screened for potential risk of adverse reactions to transcranial magnetic stimulation (TMS) by using the TMS adult safety screen (Keel et al., 2001). All subjects gave their written informed consent.

\section{Experiment 1}

Experiment setup and protocol. Subjects were seated in a relaxed position with their right hand placed on a hand pad $30 \mathrm{~cm}$ away from a carousel device (Fig. 1A). This device could present one of four different plastic drinking glasses of identical shape ( $5 \mathrm{~g}$ weight, $50 \mathrm{~mm}$ diameter, and 10 $\mathrm{cm}$ tall) but containing different weights ( $2 \times$ "light," $5 \mathrm{~g} ; 2 \times$ "heavy," $300 \mathrm{~g}$ ). One pair of glasses was opaque so that subjects could not deduce their weight, and the others were transparent. The carousel was viewed through a screen, which was either opaque or transparent. The screen was placed in front of the subject to block the subject's view while the carousel rotated between trials to present, in pseudorandom order, the next glass (Davare et al., 2009, 2010). Once the carousel stopped and the screen turned transparent ("go" signal), the subjects had to initiate, at their own pace, a precision grip of the glass using only the right thumb and the index finger and to lift it a few centimeters above the carousel. The room was kept dark except for a small headlamp that illuminated the carousel and the objects. This was to ensure that subjects were not distracted or affected by any other external cues.

The objects were presented randomly in six blocks of 40 trials. Each particular condition (TMS timing $\times$ object; see below) was repeated 20 times.

Transcranial magnetic stimulation. We used a custom-made figure-ofeight coil ( $9 \mathrm{~cm}$ outer diameter) connected to a single-pulse monophasic Magstim model 200 stimulator (Magstim Company). The stimulus was delivered over M1, with posterior to anterior induced current, through a coil held perpendicularly to the central sulcus with the handle pointing backwards. To target M1, the coil was positioned over the left motor cortex at the site in which the MEP amplitude was the greatest in both first dorsal interosseous (1DI) and abductor pollicis brevis (APB) muscles. A single TMS pulse [ $120 \%$ of the resting motor threshold (Rossini et al., 1994)] was delivered at different times during preparation for grasp but before grasp itself, i.e., at 50,100, or $150 \mathrm{~ms}$ after object presentation. The average resting motor threshold of all the subjects was $40 \pm 6 \%$ of the maximal stimulator output.

Data acquisition and analysis. The Magstim stimulator was triggered using Spike2 software and a Power 1401 CED data acquisition interface (Cambridge Electronic Design). Electromyographic (EMG) activity was recorded with bipolar surface electrodes (belly tendon), one pair positioned over the 1DI and the other over APB. The raw EMG signals were amplified (1K) (Neurolog; Digitimer) and digitized at $5 \mathrm{kHz}$ for offline analysis.

The amplitude of the evoked MEP was measured to assess the changes in CSE during the preparation of the different grasps. To normalize the MEP data across subjects, we computed the ratio between MEPs gathered during preparation of lifts preceded by a heavy and light object $\left(\mathrm{MEP}_{\text {heavy }} / \mathrm{MEP}_{\text {light }}\right)$, with a value above 1 indicating a facilitatory effect when a heavier object preceded the lift. In addition, to assess the pure effect of visual cues, we computed the ratio between MEPs recorded during preparation of lift of a visibly heavy and a visibly light object but both preceded by an object of the same weight to cancel out the sensorimotor memory effect. A value above 1 indicated an MEP facilitation when heavy visual cues were presented.
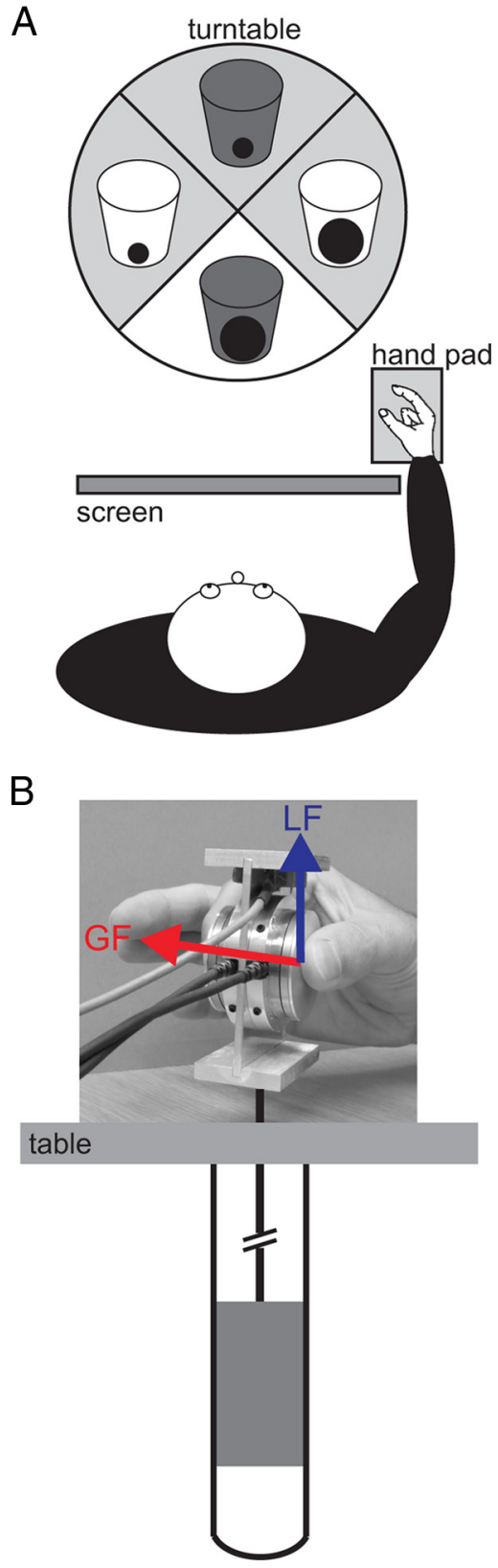

Figure 1. Schematic view of the experimental setup. $\boldsymbol{A}$, Experiment 1. The hand pad was located at $30 \mathrm{~cm}$ from the subject. The turntable randomly presented the objects $30 \mathrm{~cm}$ ahead from the hand pad. A screen, made from switchable transparent glass, was positioned between the subject and the turntable to control precisely the timing of object presentation. $B$, Experiment 2. Subjects had to grasp and lift a manipulandum with force sensors measuring the GF (red) and LF (blue). A hole under the table beneath the manipulandum contained a hollow steel cylinder. A load of $300 \mathrm{~g}$ was placed inside the cylinder and could be attached to the manipulandum to increase its weight without the subject being aware.

\section{Experiment 2}

Experiment setup. Subjects were seated comfortably with their right hand placed in a relaxed position on a table. The subjects were required to grip and lift a $225 \mathrm{~g}$ manipulandum with only their thumb and index finger (Fig. $1 B$ ). The manipulandum consisted of two parallel vertical grip surfaces of smooth aluminum ( $40 \mathrm{~mm}$ diameter, $30 \mathrm{~mm}$ apart) (similar to Davare et al., 2006). The grip surfaces covered three-dimensional forcetorque sensors (Mini $40 \mathrm{~F} / \mathrm{T}$ transducers; ATI Industrial Automation). Each sensor measured the three orthogonal forces $\left(F_{x}, F_{y}, F_{z}\right)$ along the corresponding axes intersecting the center of the grip surface. The lift force tangential to the grip surface [load force (LF)] was given by $F_{y}$. The force normal to the grip surface [grip force $(\mathrm{GF})$ ] was given by $F_{z}$. A hole 
was drilled into the table beneath the manipulandum to contain a hollow steel cylinder (Fig. $1 \mathrm{~B})$. A load of $300 \mathrm{~g}$ was placed inside the cylinder and could be attached to the manipulandum to increase its weight without the subject being aware that the load had been changed until they lifted the manipulandum. TMS was applied every $10 \mathrm{~s}$ (jitter $1.5 \mathrm{~s}$ ) while subjects were with their hand at rest, $2 \mathrm{~cm}$ in front of the manipulandum. TMS was used as the go signal. Subjects had to lift the manipulandum to a height of $5 \mathrm{~cm}$ at their own pace and replace it back to its original position after $2 \mathrm{~s}$. Talcum powder was applied to the subjects' thumb and index finger to keep the friction between the grip surfaces and fingers constant.

Experimental procedure. Subjects performed four blocks of 21 trials. In half of the blocks, TMS was applied as a sham, by placing the coil orthogonal to the scalp, to control for any disruptive effect of TMS on the grip force scaling. Within blocks, the 21 trials were presented in a pseudorandom order to have five of each transition between weights, i.e., light-after-light, heavy-after-light, heavy-after-heavy, light-afterheavy. Overall, each weight transition was repeated 10 times.

Data acquisition and analysis. The GF and LF of the thumb and index finger were digitized using the CED Power 1401 interface. For each trial, we measured the peak GF rate, given by computing the first derivative of GF using Matlab (MathWorks). Surface electrodes were also placed at subject's 1DI and APB to measure the MEPs and EMG activity during each trial. Signal 3 (Cambridge Electronic Design) was used to record and measure the MEPs and fingertip forces during each trial.

\section{Statistical analysis}

In experiment 1 , the actual MEP amplitudes were analyzed by using two-way repeated measure ANOVAs (ANOVA $A_{\mathrm{RM}}$ ), performed separately for the objects of visible weight (transparent objects) and unknown weight (opaque objects). Within-subject factors were TMS timing (50, 100, or $150 \mathrm{~ms}$ after object presentation) and previous lift weight (light or heavy). For the objects of visible weight, an additional within-subject factor was the actual visible object weight (light or heavy). Corrections for violation of sphericity were done using the Greenhouse-Geisser correction. Linear regressions were used to investigate the evolution of the CSE during the three TMS timings. In experiment 2, the MEP amplitudes and the GF scaling were analyzed using two-way ANOVA $_{\mathrm{RM}}$ with previous lift weight (light or heavy) and TMS (real or sham) as within-subject factor. Post hoc analyses were performed using Tukey's tests.

\section{Results}

\section{Experiment 1: effect of sensorimotor memory and visual} information on CSE

Effect of sensorimotor memory

The aim of experiment 1 was to investigate whether lifting objects of different weights changed the CSE. TMS was used to assess CSE at three different times before the actual grasp, i.e., 50, 100, or 150 ms after the object was presented.

A

B

C Objects of unknown weight preceded by heavy or light objects
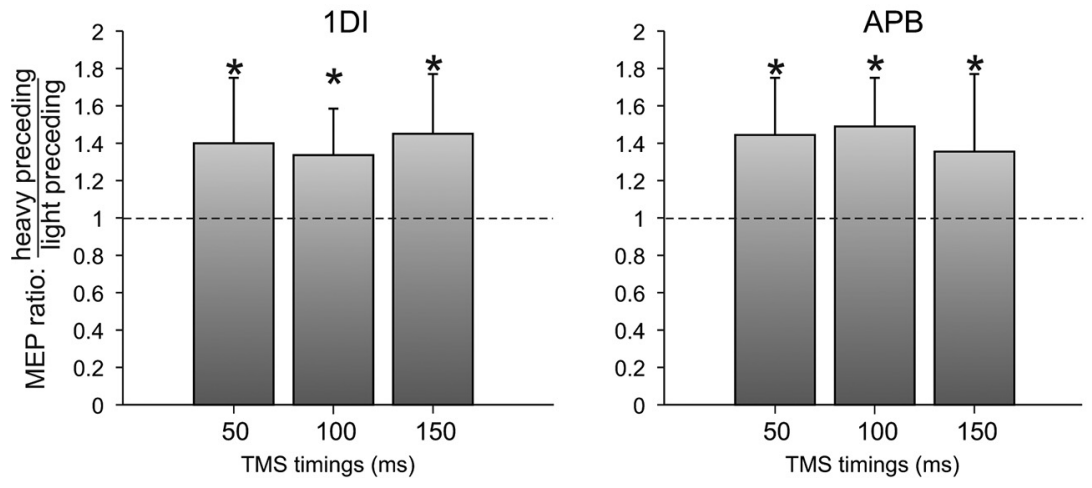
Objects of visible weight preceded by heavy or light objects

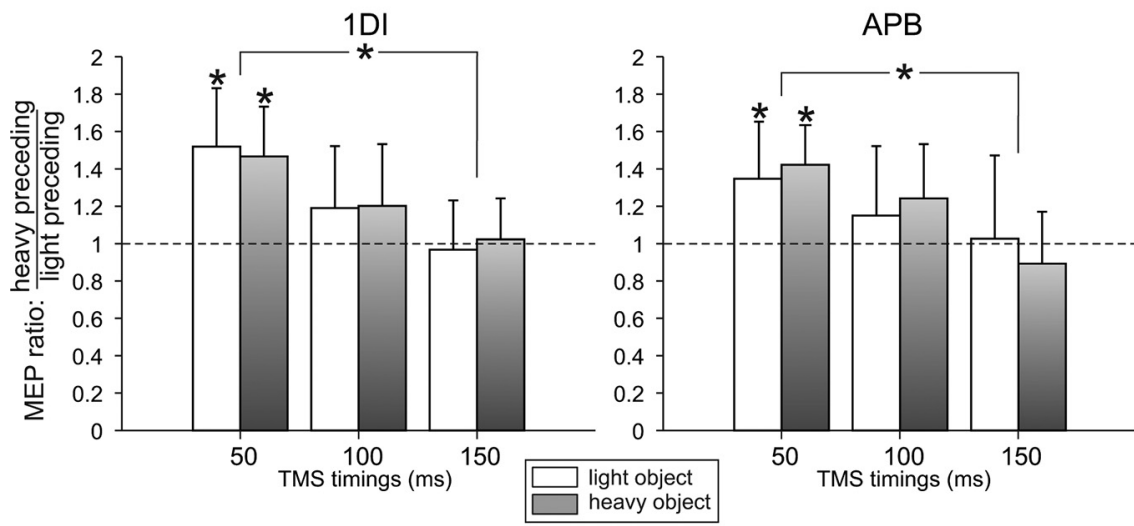
Effect of visual cues
1DI

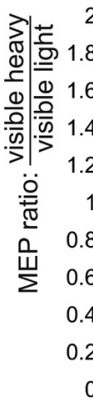

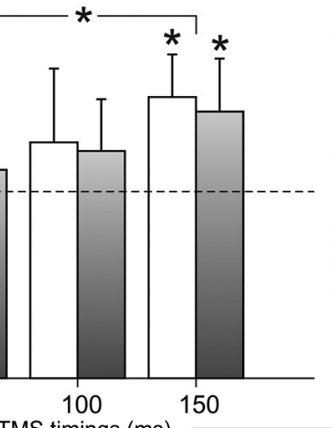

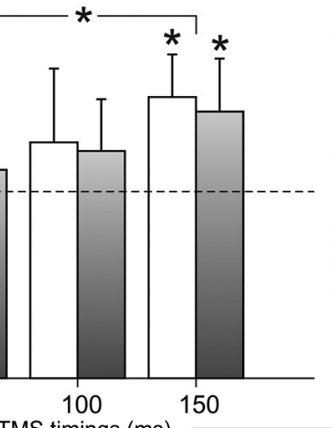

150

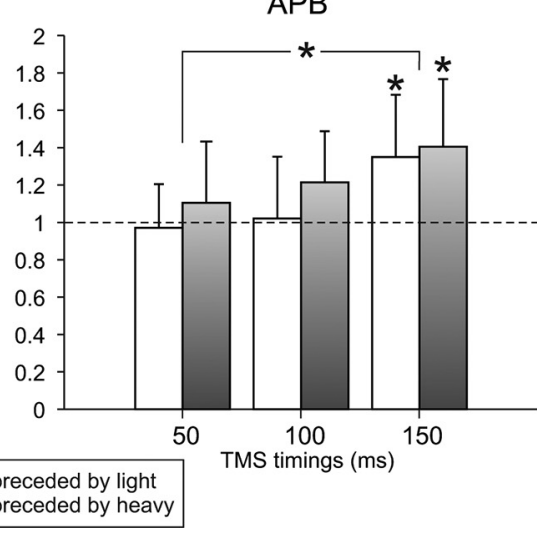

Figure 2. Corticospinal excitability during movement preparation. TMS was delivered at three timings $(x$-axis) before subjects grasped and lifted the objects. The MEP ratio ( $y$-axis) was obtained by dividing MEPs recorded when preparing lifts preceded by a heavy object by MEPs recorded when preparing a liftpreceded by a light object. $A, M E P$ ratio when preparing to lift objects of unknown weight. $B$, MEP ratio when preparing to lift objects of visible weight (light, white; heavy, gray). C, MEP ratio between MEPs recorded during preparation of lift of a heavy object and a light object but both preceded by an object of the same weight (light, white; heavy, gray). ${ }^{*} p 0.05$.

To understand how the sensorimotor memory of the previous lift affects the CSE, we first analyzed trials in which TMS was delivered during preparation of a lift of an object of unknown weight (opaque objects either light or heavy) (Fig. 2A). The ANOVA $_{R M}$ performed on the 1DI and APB MEP amplitudes showed a main effect of the previous lift weight (both $F>7.84$, both $p<0.029$ ) and no main effect of TMS timing (both $F<1$ ). The MEP amplitudes were significantly larger when the object presented was preceded by a heavy one (mean of $40 \%$; all $p<$ 0.034). Because there was no effect of TMS timing on the MEP 

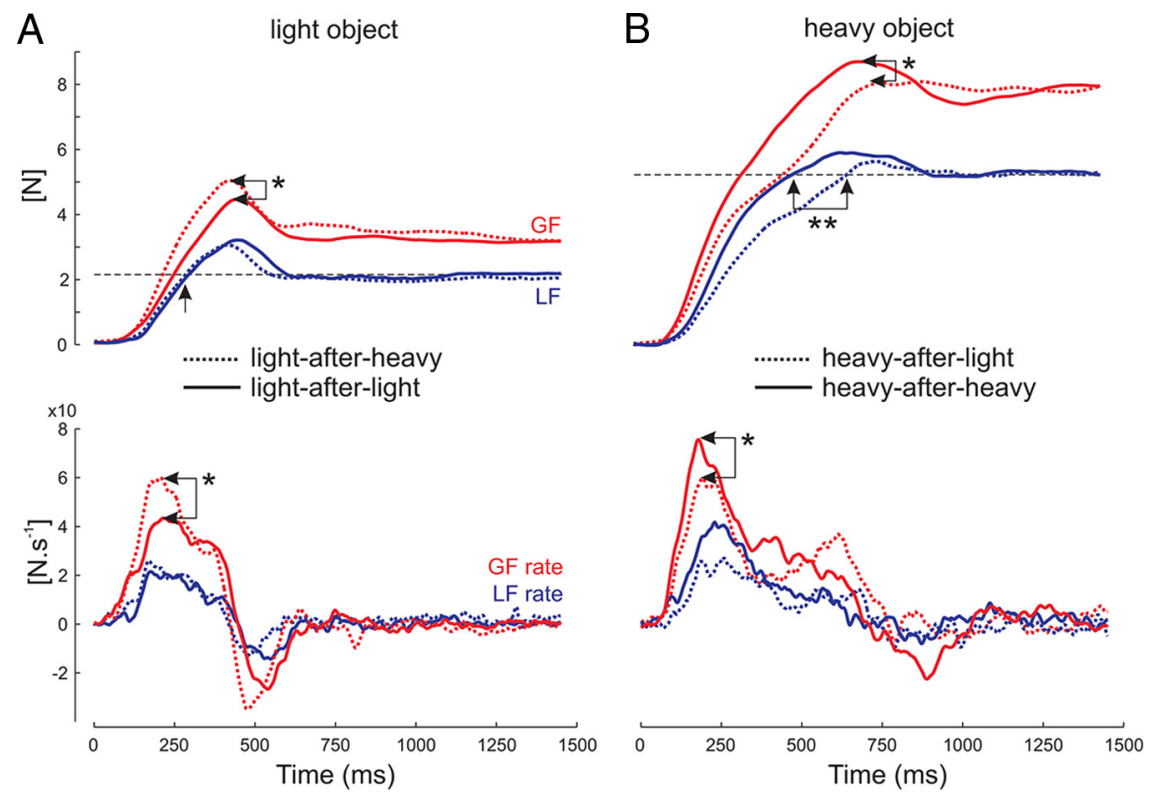

Figure 3. Typical traces for lift of a light $(\boldsymbol{A})$ and a heavy $(\boldsymbol{B})$ object. Traces show the evolution of $\mathrm{GF}$ and $\mathrm{LF}$ (top traces) and of their derivatives (GF and LF rates; bottom traces). $\boldsymbol{A}$, When lifting a light object after having picked up a heavy one (dotted lines), the GF peak and GF rate are higher than if the previous object was a light one (solid lines, see arrows *). $\boldsymbol{B}$, When lifting a heavy object after having picked up a light one (dotted lines), the GF peak and GF rate are lower (see arrows *) and the loading phase longer (see arrows ${ }^{* *}$ ) than if the previous object was a heavy one (solid lines). The horizontal dashed line represents the load of the light $(\boldsymbol{A})$ and heavy $(\boldsymbol{B})$ objects.

amplitude, this could indicate that the influence of sensorimotor memory from the previous trial remained constant across preparation of the upcoming lift of an unknown weight.

\section{Interaction between the sensorimotor memory and visual information}

To understand how visual information about the weight of an object interacts with the sensorimotor memory, we analyzed trials in which TMS was applied during preparation of lifts of objects that have their content clearly presented to subjects enabling them to determine whether it is light or heavy. Interestingly, we still found an effect of the weight of the previous lift on the MEP size when the upcoming object weight was cued by vision, but this effect was restricted to TMS applied $50 \mathrm{~ms}$ after object presentation (Fig. $2 \mathrm{~B}$ ). Indeed, the $\mathrm{ANOVA}_{\mathrm{RM}}$ showed an interaction between TMS timing and previous lift weight (ANO$\mathrm{VA}_{\mathrm{RM}}$ on 1DI and APB MEPs: both $F>5.31$, both $p<0.021$ ). Post hoc tests disclosed that the 1DI and APB MEP amplitudes were larger when the previous lift was heavy and when TMS was applied $50 \mathrm{~ms}$ after object presentation (all $p<0.031$ ). However, when TMS was applied 100 and $150 \mathrm{~ms}$ after object presentation, the sensorimotor memory effect of the previous lift was suppressed (all $p>0.137$ ) (Fig. $2 B$ ). There was a significant difference of the previous weight effect between the 50 and $150 \mathrm{~ms}$ TMS timings (both $p<0.017$ ) but not between the 50 and $100 \mathrm{~ms}$ TMS timings (both $p>0.05$ ). However, a linear regression performed with each value (50, 100, and $150 \mathrm{~ms}$ TMS timings) of all eight subjects showed a significant negative slope between the MEP amplitudes and the three TMS timings (both $p<0.003$ ).

This suggests that the visual information about the weight of an object interacts with the sensorimotor memory within M1 as soon as $100 \mathrm{~ms}$ after being presented. By $150 \mathrm{~ms}$ after information cueing the correct weight of an object is made available, the motor system has made a rapid switch from the previously used internal predictive model to the one cued by available sensory information.

\section{Effect of visual information}

To assess the simple effect of visual weight cues, we analyzed lifts performed on either visible heavy or light objects but both preceded by a lift of objects having the same weight to cancel out the sensorimotor memory effect. We found that visual information strongly facilitated CSE 150 ms after object presentation when a heavy object was presented compared with a light (all $p<0.007$ ) (Fig. 2C). Heavy visual cues did not significantly increase CSE $100 \mathrm{~ms}$ after object presentation; however, a linear regression showed a significant positive slope between the MEP sizes gathered at each TMS timing (both $p<0.008)$. This indicates that visual information about weight influences the CSE but only after it becomes available to the corticospinal system.

\section{Experiment 2: relationship between the CSE and the grip force scaling}

Experiment 2 was designed to test whether the increased CSE when lifts were preceded by heavier objects actually corresponded to the planning of a higher grip force scaling. To do so, subjects were required to lift a manipulandum without any external cue about its weight. The weight was changed in a pseudorandom order within a block without the subjects' knowledge.

When the current lift was preceded by the heavier weight, the GF was applied at a higher rate than if it was preceded by the same light object (Fig. $3 A$, arrows ${ }^{*}$ ). Regarding the effect of a lighter weight in the previous lift, the force generation followed a course similar to that of the foregoing lift (low GF rate) (Fig. 3B, arrows ${ }^{*}$ ) until the point where the object would have started to move with the previous weight. Because of the absence of movement, forces continued to increase (Fig. $3 B$, arrow ${ }^{\star *}$ ) but still at a low rate, until the load was overcome (Fig. $3 B$ ). This is consistent with previous findings (Johansson and Westling, 1988).

To analyze these effects, which involved four possible weight transitions (light-after-light, heavy-after-light, heavy-after-heavy, and light-after-heavy), we computed two different ratios to describe how subjects scaled their GF and CSE depending on the sensorimotor memory. The first ratio is given by dividing the values of GF and CSE in light-after-heavy trials by the values in light-after-light trials; a ratio above 1 indicated a facilitatory effect of a heavy preceding a light object compared with a light object. The second ratio is calculated by dividing GF and CSE values in heavy-after-heavy trials by the values in heavy-after-light trials; here a ratio below 1 signifies an inhibitory effect of a light preceding a heavy object compared with a heavy object.

When analyzing the GF rate, the performance in the four different weight transitions was consistent across all seven subjects. There was a $20 \%$ increase in the GF rate for light-after-heavy compared with light-after-light (ratio of $1.21 \pm 0.12$, mean \pm SD; $n=7 ; p=0.008$ ) and a $20 \%$ decrease in the GF rate for heavyafter-light compared with heavy-after-heavy (ratio of $0.78 \pm$ 0.09 , mean $\pm \mathrm{SD} ; n=7 ; p=0.024$ ). This finding corroborates 

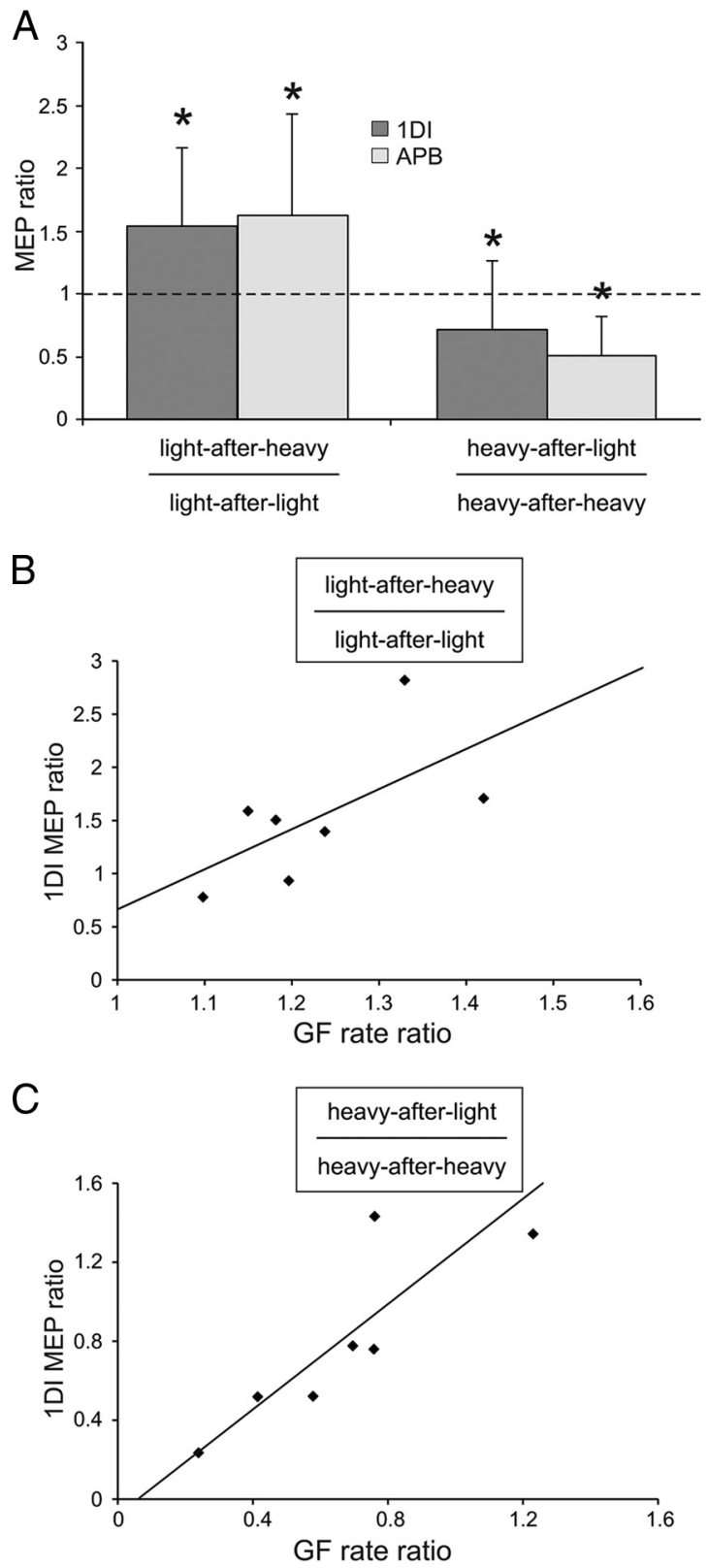

Figure 4. Relationship between the corticospinal excitability and the grip force. $A$, The $y$-axis represents the MEP ratio between lifts of a light object preceded by a heavy and a light object (left bars) and the MEP ratio between lifts of a heavy object preceded by a light and a heavy object (right bars). $\boldsymbol{B}, \boldsymbol{C}$, Correlations between the MEPs and the GF rate ratios for light $(\boldsymbol{B})$ and heavy $(\boldsymbol{C})$ objects. Note that the greater the MEP facilitation, the higher the GF rate. ${ }^{*} p<0.05$.

results from previous studies (Johansson and Westling, 1988). It is noteworthy that delivering TMS had no effect on the grip force scaling [ANOVA $\mathrm{RM}_{\mathrm{RM}}$, TMS main effect (real vs sham), $F<1$ ].

In addition, we also looked at the CSE during the preparation of the subsequent lift (Fig. $4 A$ ). The CSE data replicated the results of experiment 1 and paralleled the results of the GF rate. The mean MEP amplitude ratio showed an increase in the MEP size for light-after-heavy lifts compared with light-after-light ( $p=$ 0.003 ) and a decrease in MEP amplitude for heavy-after-light lifts compared with heavy-after-heavy $(p=0.012)$. Interestingly, we found a correlation between the GF rate ratio and the MEP facilitation in both muscles (Fig. $4 B, C$ only shows 1 DI values; light object, $r=0.74, p=0.004$; heavy object, $r=0.82, p=0.001$ ). This might indicate that the more a previous heavier lift had a facilitatory effect on the MEP size during movement preparation, the more the GF rate reached higher levels in the subsequent lift.

\section{Discussion}

The present study was designed to investigate the presence of a sensorimotor memory effect on the corticospinal system when lifting objects of different weight. In addition, we sought to determine how visual information, when it becomes available, interacted with this sensorimotor memory. To do so, we analyzed (1) the simple effect of sensorimotor memory by comparing the MEPs of lifts of an object of unknown weight preceded either by a heavier or lighter object, (2) the simple effect of visual cues by comparing lifts of visible heavy with light objects, both preceded by lift of an object having the same weight to cancel the memory effect, and (3) the interactions between visual cues and sensorimotor memory by comparing lifts of visible objects that were preceded by either a heavy or light weight.

Our results clearly demonstrate that, when no external input about the weight of an object is available, the CSE is scaled according to the weight of the object experienced in the previous lift. Interestingly, when preparing the lift of an object of visible weight, the sensorimotor memory effect was still present early after object presentation $(50 \mathrm{~ms})$ but was gradually suppressed at $100 \mathrm{~ms}$ and completely cancelled by $150 \mathrm{~ms}$ after object presentation, as soon as visual information reached the corticospinal system. By comparing lifts with a similar "sensorimotor history," we confirmed that the effect of visual cues only occurred late (150 $\mathrm{ms}$ ) after object presentation. In a second experiment, we provided evidence that the facilitation in the MEP size when preparing a lift preceded by a heavier weight was correlated with a higher grip force rate subjects actually used to lift the object. This demonstrates that the corticospinal system is able to store an internal representation of motor outputs related to weight and that, as soon as visual cues are available, it can rapidly adapt its state to generate the most appropriate motor command, and this is reflected in the excitability level of the relevant muscle representations.

Previous studies have shown that M1 is part of a larger cortical network involved in storing a sensorimotor memory of the previous lift. Functional imaging studies indicate that a large network, including frontoparietal cortical areas and the cerebellum, is active when grasping and lifting objects of different weight (Schmitz et al., 2005; Jenmalm et al., 2006). Disrupting M1 function by repetitive TMS induces deficits in accurately scaling the GF in subsequent lifts when lifting objects of the same weight (Nowak et al., 2005) or in scaling the GF according to the preceding weight (Chouinard et al., 2005; Berner et al., 2007). Indeed, on the one hand, when objects had the same weight, delivering repetitive TMS over $\mathrm{M} 1$ led to an overshoot in the GF (Nowak et al., 2005). On the other hand, when lifting objects of different weights, GF is scaled according to the previous lift based on a trial-by-trial sensorimotor memory, that is GF is either too low when a lighter weight was expected or too high when a heavier weight was expected (Johansson and Westling, 1988). Chouinard et al. (2005) found that, after repetitive TMS, their subjects lost the ability to scale grip forces according to the immediately preceding lift. Although these studies provide a causal relationship between the processing taking place in $\mathrm{M} 1$ and the establishment of a sensorimotor memory allowing the precise scaling of the grip force, here we further demonstrate that M1 could store this sensorimotor memory by modulating CSE of the involved muscle representation, with a higher CSE level giving rise to a higher grip force rate (Fig. 4). There is evidence from previous studies of a 

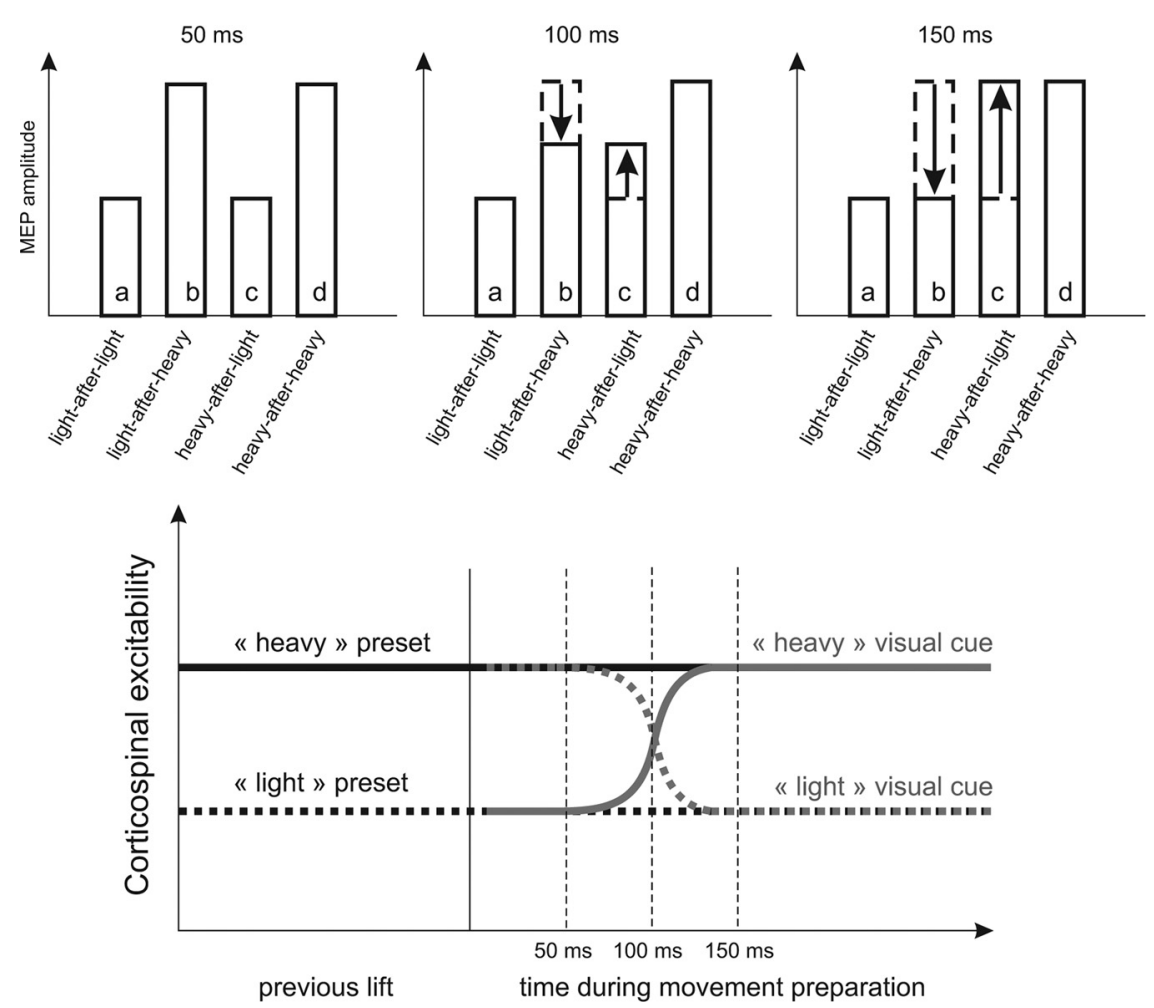

Figure 5. Interaction between information based on internal object representation and visual cues. Top, Schematic representation of the MEP amplitude for the four different lift conditions $(\boldsymbol{a}-\boldsymbol{d})$ and for each TMS timing $(50,100$, or 150 $\mathrm{ms}$ ) during movement preparation based on the data of Figure 2, B and C. At $50 \mathrm{~ms}$, the ratios between conditions light-after-heavy and light-after-light $(\boldsymbol{b} / \boldsymbol{a})$ and between heavy-after-heavy and heavy-after-light $(\boldsymbol{d} / \boldsymbol{c})$ are above 1 , meaning that $\boldsymbol{b}$ or $\boldsymbol{d}$ are greater than $\boldsymbol{a}$ or c, respectively. In contrast, ratios between heavy-after-light and light-after-light $(\boldsymbol{c} / \boldsymbol{a})$ and heavy-after-heavy and light-after-heavy $(\boldsymbol{d} / \boldsymbol{b})$ are close to 1 , meaning that MEP amplitudes in these conditions are comparable. At $150 \mathrm{~ms}$, instead, the $\boldsymbol{b} / \boldsymbol{a}$ and $\boldsymbol{d} / \mathbf{c}$ ratios are now close to 1 and the $\boldsymbol{c} / \boldsymbol{a}$ and $\boldsymbol{d} / \boldsymbol{b}$ ratios are above 1 , meaning that, whatever the previous lift, visibly heavier objects have a larger MEP than lighter ones. Intermediate values are found at $100 \mathrm{~ms}$. Bottom, Schematic evolution of the CSE during movement preparation depending on the previous lift and the upcoming visual cues. For example, if the previous object was light (black dotted line), (SE is low and remains low if the visual cues about the upcoming object are indicating a light weight (gray dotted line) or increases if cues are informative of a heavy object (gray solid line).

sensorimotor memory in M1 (Li et al., 2001; Nowak et al., 2005; Berner et al., 2007), and it is therefore plausible that the MEP changes we have documented might have originated from M1 itself. However, we cannot exclude the possibility that other descending influences may have altered levels of spinal motoneuronal excitability independent of M1 input. The cerebellum, another likely source of internal models for predictive grasp (Schmitz et al., 2005; Bursztyn et al., 2006; Jenmalm et al., 2006), may exert much of its influence through motor cortex and therefore contribute to M1 excitability changes.

In addition, we found that the motor system can make a rapid switch from a previously used internal predictive model to a different one by available sensory information. To explain how CSE is influenced by the previous weight lifted and by visual cues, we formulated a model of interactions between the stored sensorimotor memory and available visual information (Fig. 5). The top row shows schematically the raw MEP size for each object at the three TMS timings tested. At $50 \mathrm{~ms}$ after object presentation, the CSE is only influenced by the previous weight because only the ratios between objects of different weight history are above 1 (Fig. 5, b/a, d/c). Indeed, ratios between objects of similar weight history and different visible weight showed values of 1 . In contrast, $150 \mathrm{~ms}$ after object presentation, the ratios reversed and now have a ratio above 1 between objects of different visible weight (Fig. 5, $c / a, d / b$ ) but not for objects of different weight history. Transitional results were found 100 ms after visual cues are presented. It is noteworthy that, when preparing the lift of a visible light object that was preceded by a heavier one, the effect of the light visual cue decreased CSE. Conversely, preparing the lift of a heavy object preceded by a lighter one yielded the heavy visual information to increase CSE. The evolution of CSE is depicted in the bottom of Figure 5. As soon as visual cues are available to the motor system, the CSE gradually moves from its anterior state, defined by the previous weight, to a state that corresponds to the visual information. The fact that visual information only interacts with CSE after 100-150 ms is in agreement with previous studies. Visual cues for movement reach human premotor areas at $\sim 100 \mathrm{~ms}$ (Terao et al., 1998; Schluter et al., 1999). In a recent study, Prabhu et al. (2007) reported that visual object cues could not influence CSE before $150 \mathrm{~ms}$ after object presentation. In monkeys, it has been reported that, on a visual conditional task, the mean onset of signal-related units in the premotor cortex was $\sim 140 \mathrm{~ms}$ after the instruction stimuli (Johnson et al., 1996).

A predictive scaling of grip force adapted to the weight of an object can be explained within the theoretical framework of internal forward models (Wolpert and Flanagan, 2001). Indeed, when lifting an object, the subject's own upward movement causes the load force to increase, and, to prevent slippage, the grip force has to be increased in a parallel manner (Flanagan and Wing, 1997). Because of the inevitable delays in cutaneous afferent pathways (Johansson and Westling, 1984), this predictive modulation of the grip force cannot be based on peripheral feedback. Therefore, anticipatory grip force increases must be generated by using a predictive model of the consequences of the action (Flanagan and Wing, 1997; Blakemore et al., 1998). Our results suggest that this predictive internal model sets a sensorimotor memory in M1 by modulating the CSE. Interestingly, Quaney et al. (2003) found that this sensorimotor memory was not specific for object lifting, which suggests that previous actions bias M1 outputs even if they are not related to the present action. This could be a more ecological way for the motor system to learn actions in a stochastic environment (Quaney et al., 2003). In our present experiment, one strategy could be to generate a force midway between the two possible weights. However, this would require the motor system to rescale the motor output too often. Therefore, we suggest that a strategy based on the previous action, as used by subjects in the present experiment, is more optimal.

In conclusion, we provide evidence that M1 stores a sensorimotor memory of the weight of an object by changing the level of excitability of the involved muscle representations. If visual in- 
formation becomes available, it is then used rapidly to switch between different models of predictive grasp control.

\section{References}

Berner J, Schönfeldt-Lecuona C, Nowak DA (2007) Sensorimotor memory for fingertip forces during object lifting: the role of the primary motor cortex. Neuropsychologia 45:1931-1938.

Blakemore SJ, Goodbody SJ, Wolpert DM (1998) Predicting the consequences of our own actions: the role of sensorimotor context estimation. J Neurosci 18:7511-7518.

Bursztyn LL, Ganesh G, Imamizu H, Kawato M, Flanagan JR (2006) Neural correlates of internal-model loading. Curr Biol 16:2440-2445.

Chouinard PA, Leonard G, Paus T (2005) Role of the primary motor and dorsal premotor cortices in the anticipation of forces during object lifting. J Neurosci 25:2277-2284.

Davare M, Andres M, Cosnard G, Thonnard JL, Olivier E (2006) Dissociating the role of ventral and dorsal premotor cortex in precision grasping. J Neurosci 26:2260-2268.

Davare M, Montague K, Olivier E, Rothwell JC, Lemon RN (2009) Ventral premotor to primary motor cortical interactions during object-driven grasp in humans. Cortex 45:1050-1057.

Davare M, Rothwell JC, Lemon RN (2010) Causal connectivity between the human anterior intraparietal area and premotor cortex during grasp. Curr Biol 20:176-181.

Flanagan JR, Wing AM (1997) The role of internal models in motion planning and control: evidence from grip force adjustments during movements of hand-held loads. J Neurosci 17:1519-1528.

Gordon AM, Forssberg H, Johansson RS, Westling G (1991) Visual size cues in the programming of manipulative forces during precision grip. Exp Brain Res 83:477-482.

Jenmalm P, Johansson RS (1997) Visual and somatosensory information about object shape control manipulative fingertip forces. J Neurosci 17:4486-4499.

Jenmalm P, Schmitz C, Forssberg H, Ehrsson HH (2006) Lighter or heavier than predicted: neural correlates of corrective mechanisms during erroneously programmed lifts. J Neurosci 26:9015-9021.

Johansson RS, Westling G (1984) Roles of glabrous skin receptors and sensorimotor memory in automatic control of precision grip when lifting rougher or more slippery objects. Exp Brain Res 56:550-564.

Johansson RS, Westling G (1988) Coordinated isometric muscle commands adequately and erroneously programmed for the weight during lifting task with precision grip. Exp Brain Res 71:59-71.

Johnson PB, Ferraina S, Bianchi L, Caminiti R (1996) Cortical networks for visual reaching: physiological and anatomical organization of frontal and parietal lobe arm regions. Cereb Cortex 6:102-119.

Keel JC, Smith MJ, Wassermann EM (2001) A safety screening questionnaire for transcranial magnetic stimulation. Clin Neurophysiol 112:720.

Li CS, Padoa-Schioppa C, Bizzi E (2001) Neuronal correlates of motor performance and motor learning in the primary motor cortex of monkeys adapting to an external force field. Neuron 30:593-607.

Nowak DA, Voss M, Huang YZ, Wolpert DM, Rothwell JC (2005) Highfrequency repetitive transcranial magnetic stimulation over the hand area of the primary motor cortex disturbs predictive grip force scaling. Eur J Neurosci 22:2392-2396.

Oldfield RC (1971) The assessment and analysis of handedness: the Edinburgh inventory. Neuropsychologia 9:97-113.

Prabhu G, Voss M, Brochier T, Cattaneo L, Haggard P, Lemon R (2007) Excitability of human motor cortex inputs prior to grasp. J Physiol 581:189-201.

Quaney BM, Rotella DL, Peterson C, Cole KJ (2003) Sensorimotor memory for fingertip forces: evidence for a task-independent motor memory. J Neurosci 23:1981-1986.

Rossini PM, Barker AT, Berardelli A, Caramia MD, Caruso G, Cracco RQ, Dimitrijević MR, Hallett M, Katayama Y, Lücking CH (1994) Noninvasive electrical and magnetic stimulation of the brain, spinal cord and roots: basic principles and procedures for routine clinical application. Report of an IFCN committee. Electroencephalogr Clin Neurophysiol 91:79-92.

Schluter ND, Rushworth MF, Mills KR, Passingham RE (1999) Signal-, set-, and movement-related activity in the human premotor cortex. Neuropsychologia 37:233-243.

Schmitz C, Jenmalm P, Ehrsson HH, Forssberg H (2005) Brain activity during predictable and unpredictable weight changes when lifting objects. J Neurophysiol 93:1498-1509.

Terao Y, Fukuda H, Ugawa Y, Hikosaka O, Hanajima R, Furubayashi T, Sakai K, Miyauchi S, Sasaki Y, Kanazawa I (1998) Visualization of the information flow through human oculomotor cortical regions by transcranial magnetic stimulation. J Neurophysiol 80:936-946.

Witney AG, Goodbody SJ, Wolpert DM (2000) Learning and decay of prediction in object manipulation. J Neurophysiol 84:334-343.

Wolpert DM, Flanagan JR (2001) Motor prediction. Curr Biol 11:R729R732. 